\title{
Comparison of the Orders of Gas-Phase Basicities and Ammonium Ion Affinities of Polyethers by the Kinetic Method and Ligand Exchange Technique
}

\author{
H.-F. Wu and J. S. Brodbelt \\ Department of Chemistry and Biochemistry, University of Texas, Austin, Texas, USA
}

\begin{abstract}
The orders of relative gas-phase basicities and ammonium ion affinities of a series of polyethers obtained by application of the kinetic method and ligand exchange technique are compared to evaluate the discrepancies of results between the two techniques. The order of gas-phase basicities determined by the ligand exchange technique in a quadrupole ion trap agrees with the order established previously by Kebarle using equilibritum methods in a high-pressure mass spectrometer. The order obtained by the kinetic method in a triple quadrupole mass spectrometer varies for the ranking of one polyether (12-crown-4), and this discrepancy is attributed to a difference in the rates of the competing dissociation pathways from the triethylene glycol dimethyl ether/12-crown-4 proton-bound adduct, owing to a substantial variation in the flexibilities of these two ethers. For the order of gas-phase ammonium ion affinities, the kinetic method results agree overall with the ligand exchange results; however, the order of ammonium ion affinities for tetraethylene glycol dimethyl ether and 15-crown-5 could not be differentiated by the ligand exchange method because of the rapidity of ammonium ion transfer between the two polyethers in both directions. (/ Am Soc Mass Spectrom 1993, 4, 718-722)
\end{abstract}

$\mathrm{O}$ ngoing advances in the development of mass spectrometric instrumentation have stimulated activity in several areas of gas-phase ion chemistry related to the measurement of ion binding affinities. In many recent cases, such studies involve measurements for complex involatile molecules or involve a diverse array of binding ions, including metals, clusters, or solvated cations. For example, the implementation of fast-atom bombardment [1], laser desorption [2], and electrospray ionization [3] methods has promoted growth in the field of biologic mass spectrometry [4]. As a result, there have been efforts by several groups [5] to evaluate the gas-phase basicities of biologic molecules because of the importance of this thermodynamic parameter in terms of understanding sites of ionization and their relationship to dissociation pathways. Note that the gas-phase basicity of a molecule is defined as $-\Delta G$ for its protonation reaction, whereas the proton affinity is $-\Delta H$ for the same reaction. The differentiating factor is the temperaturedependent entropy term. There has also been recent interest in the evaluation of host-guest complexation in the gas phase $[6,7]$ to understand the nature of the

Address reprint requests to Jennifer Brodbelt, Department of Chemistry and Biochemistry, University of Texas, Austin, TX 78712-1167. intrinsic binding interactions involved in molecular recognition [8]. Thus, several groups have investigated the relative binding affinities of model hosts, such as crown ethers, to model guests, such as alkali metal ions [6] and ammonium ions [7].

Often the popular equilibrium methods are not viable for measurement of ion binding affinities owing to the inability to precisely control or monitor the gasphase concentrations of analytes, as in laser-desorbed species. Thus, two alternative techniques have been commonly applied for such measurements: the kinetic method [9] and the ligand exchange and/or bracketing technique [10-13]. The kinetic method was first described for the determination of relative gas-phase basicities of simple organic molecules [9]. In this method, a proton-bound complex of two different bases is formed by ion-molecule reactions in the gas phase; then the complex is typically collisionally activated to promote dissociation. Cleavage at the proton bridge results in competitive formation of each protonated base involved in the complex, and the ratio of the abundances is then related to the proton affinity of each base. Because the two substrates involved in the complex must necessarily have similar structures to avoid the influence of unequal entropy factors on the dissociation kinetics, it is often assumed that the relative proton affinities measured by the kinetic method 
are thus equivalent to their relative gas-phase basicities (i.e., $\Delta S=0$ ).

In contrast, the ligand exchange or bracketing technique is a method in which a cation (i.e., proton, metal ion) is allowed to transfer between two bases in the gas phase [12-14]. For example, one cationized base is permitted to interact with a second neutral base, and observation of the transfer of the cation to the second base indicates that the second base has a higher intrinsic cation affinity. Typically, the cation transfer reaction is observed in both directions to confirm the order of affinities. Originally, this method was typically performed in a relatively high-pressure ion source, and the total pressure of reactants admitted was varied systematically to observe the change in product ion distribution and thus determine the favored direction of the cation transfer reaction [12, 13]. In recent years, ion trapping techniques have often been used to undertake this type of experiment [14], with the added advantages that the cation transfer reactions can be monitored as a function of time, and one reactant ion may be isolated and allowed to selectively react with the second reactant neutral. This latter feature allows determination of affinities without requiring the ability to uniformly vary the total reactant pressure; however, the neutral gas-phase concentrations of each base must be controlled in some way to ensure nearly equivalent or at least well-characterized collision probabilities between the two bases, allowing accurate extraction of thermodynamic data. In general, both the kinetic method and the bracketing technique, in contrast to equilibrium methods, allow estimation of relative ion affinities for involatile molecules and cases that may involve unusual cation binding studies. Additionally, these methods can be used either to generate numerical values of ion affinities if appropriate reference compounds with known ion affinities are available or to merely establish trends in ion affinities for series of compounds.

The use of the kinetic method for measurements involving more complex molecules, such as those that might promote multiple binding interactions to a cation, has generated some controversy lately because of the known but poorly understood shortcornings with regard to entropic factors. One of the main assumptions underlying this technique is that the frequency factors for each competing dissociation pathway of the cation-bound complex are nearly identical, and thus it has been accepted that the structures of the two molecules involved in the complex must be similar so that entropic discrepancies may be neglected. If the two dissociation pathways of the cation-bound adduct have substantially different rates due to entropic contributions, then the measured ion abundances for the fragment ions will not accurately reflect the relative affinities of each molecule. Likewise, the bracketing technique is only effective if one can approximate the gas-phase concentrations of each reactant so that the influence of the competing kinetics of ligand exchange can be evaluated with respect to the resulting observed product distributions. In fact, there have already been some reported discrepancies for the gas-phase basicities of small peptides measured by the kinetic method and by bracketing techniques [5].

Because of the concerns described above, and because of our own use of the kinetic method to measure orders of relative binding affinities in host-guest chemistry, we recently undertook a comparative study of the kinetic method and the ligand exchange technique for a relatively complex experimental case in which both the reactants of interest and the binding cation offer potential problems. It is generally assumed that the same types of binding forces, although differing in magnitude, that participate in formation of a single cation-attached molecule will also operate in a cation-bound complex consisting of two different molecules, and thus a comparison of the kinetic method and the bracketing technique is a rational undertaking. This assumption appears reasonable because any cation transfer reaction for a bracketing experiment must proceed through a state in which both ligands are transiently bound to the cation in some fashion, somewhat analogous to the kinetic method in which both ligands are bound to a single cation.

The system of interest in the present study involves a series of polyethers binding the proton and the ammonium ion. The polyethers, including several polyethylene glycols, polyethylene glycol dimethyl ethers (glymes), and crown ethers, have been commonly used as model hosts in the field of molecular recognition [8] because they have multiple binding sites and thus presumably participate in multisite interactions in the gas phase as well. The proton is the most charge-dense and smallest cation available, whereas the ammonium ion is a bulky tetrahedral cation that may promote multisite coordination. Thus, these two types of cations should allow some meaningful insight into how the size and charge distribution of the cation affect the outcome of the binding studies. Likewise, the choice of polyethers as model ligands permits an evaluation of the kinetic method and ligand exchange technique for determination of binding affinities for large molecules with many binding sites. The objective is to examine the severity of discrepancies in the relative orders of cation affinities as measured by these two methods.

\section{Experimental}

Two types of mass spectrometers were used for this study. A Finnigan TSO70 triple quadrupole mass spectrometer equipped with a chemical ionization source was used to measure the cation affinities of the polyethers by the kinetic method. A Finnigan ITMS ion trap mass spectrometer was used to measure the affinities by the ligand exchange technique.

The determination of the order of gas-phase basicities and ammonium ion affinities of polyethers by the 
kinetic method has been reported previously by our laboratory [7], and details of the experimental method have been described previously [7]. The samples were introduced by a direct insertion probe, and typical sample pressure was $1-3 \times 10^{-6}$ torr. Ammonia was admitted to the source to 2 torr as a chemical ionization agent. The relatively high pressure of the chemical ionization source ensures that the complexes experience hundreds of collisions, and thus thermal equilibrium conditions are approached. The desired precursor ion was selected with the first quadrupole and passed into the collision quadrupole at an average collision energy of $2 \mathrm{eV}$. Typical collision gas pressure was 0.6 mtorr of argon. For the compounds of interest in this study, the ligand exchange method cannot be performed with this type of spectrometer because the total pressure of reactant polyethers cannot be routinely and consistently varied owing to the low volatility of the larger polyethers.

For the ion trap experiments, two polyethers were admitted into the vacuum chamber to approximately $2 \times 10^{-6}$ torr. Ammonia was admitted to nominally $3 \times 10^{-6}$ torr, and 1 mtorr helium was used as the buffer gas. An electron ionization interval of $5 \mathrm{~ms}$ was used to allow formation of ammonium ions, protonated molecules, and $\left[\mathrm{M}_{n}+\mathrm{NH}_{4}\right]^{+}$ions. The $\left[\mathrm{M}_{n}+\right.$ $\mathrm{H}]^{+}$or $\left[\mathrm{M}_{n}+\mathrm{NH}_{4}\right]^{+}$ion was apex isolated with a combination of direct current and radiofrequency (RF) voltages, and then allowed to react for 0-500 ms with polyether neutrals present in the trap. The resulting product ions were mass analyzed by using the massselective instability mode of operation. The kinetic method could not be used in the ion trap because of the inability to form sufficient abundances of cationbound adducts.

All compounds except 21-crown-7 were obtained from Aldrich Chemical Co. (Milwaukee, WI) and used without further purification. The 21-crown-7 was obtained from Parish Chemical Co. (Orem, UT). Purities were greater than $97 \%$.

\section{Results and Discussion}

The relative orders of ammonium ion affinities and gas-phase basicities as measured by the kinetic method in a triple quadrupole mass spectrometer have been reported previously [7], but are summarized again in Table 1 to facilitate the present comparison. Gas-phase basicities have been previously measured for several crowns and glyme analogs by equilibrium methods in a pulsed high-pressure mass spectrometer [15]. The order of affinities reported in that earlier work agrees with the order established in Table 1, with one discrepancy. The gas-phase basicity measured by the equilibrium method for 12 -crown -4 is $0.5 \mathrm{kcal} / \mathrm{mol}$ higher than the gas-phase basicity of triglyme [15], although the proton affinity estimated for 12-crown-4 is actually $3 \mathrm{kcal} / \mathrm{mol}$ lower than that of triglyme [15]. Thus, there is a $3.5-\mathrm{kcal} / \mathrm{mol}$ difference in the relative
Table 1. Orders of relative affinities of Polyethers ${ }^{\star}$

\begin{tabular}{l|l}
\hline \multicolumn{1}{c}{$\mathrm{H}^{+}$} & $\mathrm{NH}_{4}^{+}$ \\
\hline \hline Triglycal $^{12-\text { Crown-4 }^{\mathrm{b}}}$ & Triglycol \\
Triglyme $^{\mathrm{b}}$ & 12 -Crown-4 \\
15 -Crown-5 & Triglyme \\
Tetraglyme & Tetraglycol \\
18-Crown-6 & 15 -Crown-5 \\
Tetraglycol & Tetraglyme $^{\mathrm{c}}$ \\
21-Crown-7 & 18 -Crown-6 \\
\hline
\end{tabular}

arder of increasing affinity down the column.

${ }^{b}$ The order of gas-phase basicities of these two polyethers reverses by the ligand exchange technique.

-These telative ammonium ion affinities of these two polyethers could not be differentiated by the ligand exchange technique.

affinities due to entropic factors (i.e., the $\Delta S$ term). As shown in Table 1 , the relative gas-phase basicity of 12-crown- 4 , as measured by the kinetic method, appears to be lower than that of triglyme. This result suggests a partially inadequate accounting of the entropy factors involved in the competing dissociation reactions, which is not completely unexpected in light of the large $3.5-\mathrm{kcal} / \mathrm{mol}$ difference between the gasphase basicities and proton affinities as reported by Kebarle and co-workers [15]. One possible explanation for the discrepancy is that the cleavages of hydrogen bonds between the proton and triglyme molecule within the proton-bound triglyme/12-crown-4 adduct occur at a somewhat slower rate than the hydrogen bonds between the proton and 12-crown-4 molecule, owing to the enhanced flexibility of the open-chain ether relative to the more rigid 12-crown-4. Of all the crown ethers, 12-crown-4 is clearly the most rigid and thus would be most susceptible to this accelerated bond-cleavage rate problem. There have been no other reports about the orders of gas-phase ammonium ion affinities. Thus, quantitative values of ammonium ion affinities for the polyethers are not assigned in Table 1 owing to the lack of appropriate reference values of ammonium ion affinities, which are required as calibration and anchor points.

For determination of the order of affinities by the ligand exchange technique in the quadrupole ion trap, the proton transfer and ammonium ion transfer reactions were systematically repeated for many different pairs of polyethers. An example of the proton transfer experiment is illustrated in Figure 1, and an example of the ammonium ion transfer experiment is shown in Figure 2 for the reactions between tetraethylene glycol (tetraglycol) and tetraethylene glycol dimethyl ether (tetraglyme). As seen in Figure 1, tetraglycol has a higher gas-phase basicity because it successfully deprotonates protonated tetraglyme, and the reverse reaction does not occur. In contrast, tetraglyme has a higher ammonium ion affinity because it abstracts an ammonium ion from the tetraglycol $\left[\mathrm{M}+\mathrm{NH}_{4}\right]^{+}$ion (Figure 2). In fact, the ammonium ion transfer from 

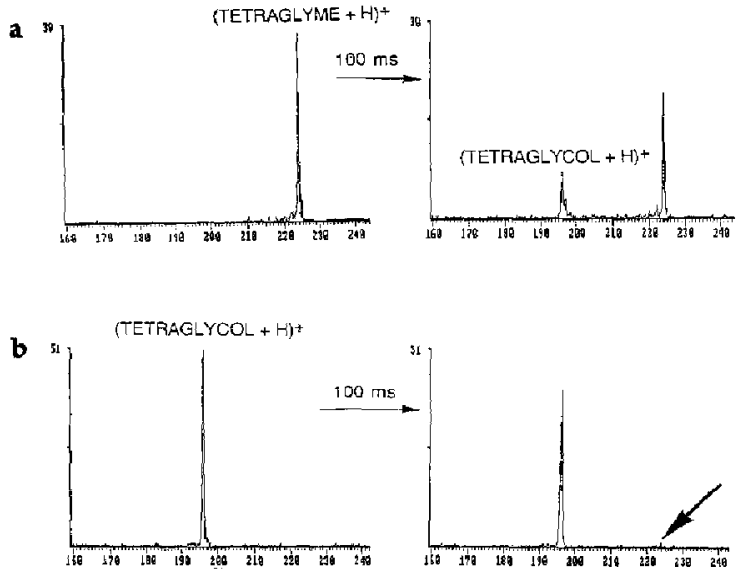

Figure 1. Proton exchange between tetraethylene glycol (tetraglycol) and tetraethylene glycol dimethyl ether (tetraglyme) in a quadrupole ion trap: (a) isolation of protonated tetraglyme, followed by a 100-ms reaction period; (b) isolation of protonated tetraglycol, followed by a $100 \mathrm{~ms}$ reaction period. Each polyether was admitted at a pressure of nominally $3 \times 10^{-6}$ torr.

tetraglycol to tetraglyme is so fast that it occurs to some extent during the analytical scan, thus producing the ion at $m / z 240$ in Figure $2 b$, shown at 0 -ms reaction time. No other products are observed during these experiments.

The complete orders of gas-phase basicities and ammonium ion affinities obtained by the ligand exchange technique in the quadrupole ion trap match the orders established by the kinetic method (Table 1), with two exceptions. First, the ammonium ion exchange reactions between 15-crown-5 and its acyclic analog, tetraglyme, occurred in both directions at similar rates, so it was difficult to distinguish the one of
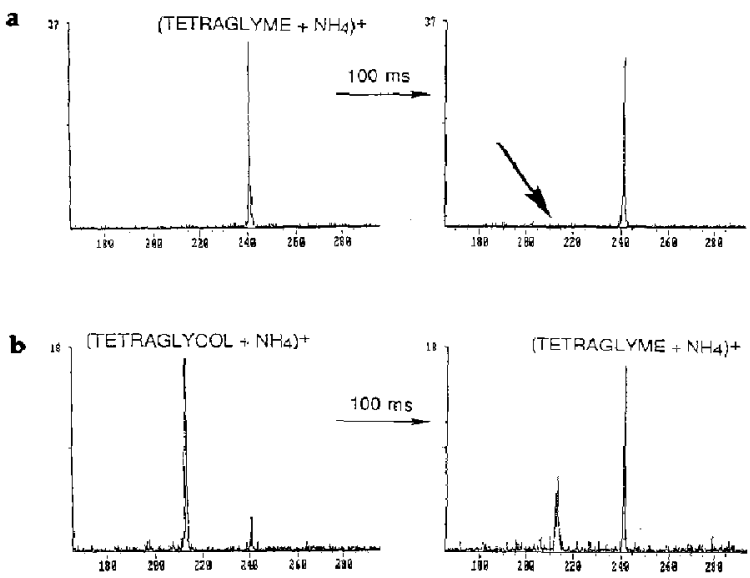

Figure 2. Ammonium ion exchange between tetraethylene glycol (tetraglycol) and tetraethylene glycol dimethyl ether (tetraglyme) in a quadrupole ion trap: (a) isolation of the [tetraglyme $\left.+\mathrm{NH}_{4}\right]^{+}$ion, followed by a 100 -ms reaction period; (b) isolation of the [tetraglycol $\left.+\mathrm{NH}_{4}\right]^{+}$ion, followed by a 100 -ms reaction period. Each polyether was admitted at a pressure of nominally $3 \times 10^{-6}$ torr higher ammonium ion affinity in the ion trap. It has recently been shown that some ions stored in a quadrupole ion trap may possess over $2 \mathrm{kcal} / \mathrm{mol}$ of kinetic energy [16], owing to the continuous acceleration and deceleration of ions trapped by an oscillating RF field, and this energy may be sufficient to drive some reactions of low endothermicity in the forward direction; however, in the present study all stored ions would be expected to be affected somewhat equally by this translational energization. The fact that the ammonium ion transfer reaction for the 15-crown-5/tetraglyme system proceeds in both directions to similar extents suggests that the ammonium ion affinities of these two polyethers are extremely close to each other. Thus, within the experimental limitations of the quadrupole ion trap, the ammonium ion affinities of these two polyethers could not be differentiated by the ligand exchange technique. By application of the kinetic method in the triple quadrupole mass spectrometer, the ammonium ion affinity of tetraglyme appears to be distinctly greater than that of 15-crown-5.

The second discrepancy involves the order of relative gas-phase basicities for 12-crown-4 and triglyme. The gas-phase basicity of 12 -crown-4 is determined to be higher than that of triglyme by the ligand exchange method in the ion trap, so this order of affinities reverses from the one shown in Table 1 for the kinetic method. In fact, this reversal causes the order of gasphase basicities of these two ethers to agree with the accepted order established in ref 15 . The proton transfer reaction occurs in both directions for this pair of polyethers, indicating that the gas-phase basicities are indeed quite similar and highlighting the important influence of kinetic factors (and thus the importance of monitoring the pressures of each reactant) on these exchange reactions.

The temperature dependence of the ligand exchange experiments in the quadrupole ion trap was not extensively evaluated owing to the inability to assign an "ion temperature" in the trapping environment. In the quadrupole ion trap, ions are continuously accelerated and decelerated during their trajectories because of the oscillating RF field, and they experience numerous activating and deactivating collisions with helium throughout their storage. Thus, although different ions experience the same type of field overall and would be expected to possess similar average amounts of kinetic energy, their average internal temperatures are not easily estimated nor easily varied by simply changing the temperature of the ion trap vacuum manifold. Fortunately, the pairs of reactants used for the ligand exchange technique will experience the same environment in the trap, and thus temperaturerelated factors may tend to diminish in importance. Moreover, due to the failings of the equilibrium technique for measurements involving involatile molecules. the use of the ligand exchange method in a quadrupole ion trap provides at least one viable means, albeit with some limitations, to estimate relative ion affinities. 


\section{Conclusions}

To obtain the best approximations of relative ion affinities, one would clearly like to perform temperature-dependent equilibrium measurements, such as could be undertaken with a high pressure mass spectrometer; however, this approach is often not possible for studies involving involatile substrates, which have ill-defined or poorly controlled vapor pressures. For the systems studied herein, the kinetic method apparently proves effective for developing relative scales of affinities for both a minuscule cation (i.e., proton) and a bulky cation (i.e., ammonium ion), which may bind through multiple hydrogen bonds. The orders of gasphase basicities and ammonium ion affinities change for the series of polyethers, and these variations are reasonably replicated by each technique. Clearly, this study does not prove the universal applicability of the kinetic method; however, it is noteworthy that it may succeed in some situations for determination of orders of affinities of ligands possessing multiple binding sites. Likewise, the ligand exchange technique may give somewhat ambiguous results for distinguishing the ion affinities of ligands that have very similar affinities. In this latter case, it is essential that the gas-phase concentrations of the two neutral ligands be identical or well defined so that reaction rates can be estimated. This constraint is increasingly difficult to maintain for larger and less volatile molecules. In conclusion, for those cases in which multiple binding sites may be involved, it appears to be advisable, when possible, to confirm the results of the kinetic method by using a second technique; but the kinetic method alone may often provide a rapid and facile method for generating reasonable qualitative orders of ion affinities.

\section{Acknowledgments}

Support from the Welch Foundation (F-1155), NIH (RO1 GM46723-01), and an ACS-PRF grant (25485-AC5) are acknowledged.

\section{References}

1. Barber, M.; Bordoli, R. J.; Elliott, G. J.; Sedgwick, R. D.; Tyler, A. N. Anal. Chem. 1982, 54,645A.

2. Lubman, D. M., Ed. Lasers and Mass Spectrometry; Oxford University Press: New York, 1990.

3. Fenn, J. B.; Mann, M.; Meng, C. K.; Wang, S. F.; Whitehous, C. M. Mass Spectrom. Rez. 1990, 9, 37.

4. McEwen, C. N.; Larsen, B. S., Eds. Mass Spectrometry of Biological Molecules; Marcel Dekker: New York, 1990.

5. (a) Gorman, G. S.; Speir, I. P.; Turner, C. A.; Amster, I. I. J. Am. Chem. Soc., 1992, 114, 3988; (b) Greco, F.; Liguori, A.; Sindona, G.; Uccella, N. J. Am. Chem. Soc. 1990, 112, 9092; (c) Bojesen, G. J. Am. Chem. Soc. 1987, 109, 5557; (d) Wu, Z.; Fenselau, C. Rapid Commun. Mass Spectrom. 1992, 6, 403; (e) Isa, K.; Omote, T.: Amaya, M. Org. Mass Spectrom. 1990, 25, 620; (f) Compbell, S.; Beauchamp, J. L.; Rempe, M,; Lichtenberger, D. L. Int. J. Mass Spectrom. Ion Processes 1992, 117, 83; (g) OHair, R. A. J.; Bowie, J. H.; Bronert, S. Int. J. Mass Spectrom. Ion Processes 1992, 117, 23.

6. (a) Brodbelt, J.; Maleknia, S, Liou, C.; Lagow, R. J. Am. Chem. Soc. 1991, 113, 5913-5914; (b) Zhang, H.; Chu, I.; Leming, S.; Dearden, D. A. I. Am. Chem. Soc. 1991, 113, 7415 7417; (c) Maleknia, S.; Brodbelt, J. J. Am. Chem. Soc., 1992, 114, 4295; (d) Liou, C.-C.; Brodbelt, J. I. Am. Soc. Mass Spectrom. 1992, 3, 543.

7. Liou, C.-C.; Brodbelt, J. J. Am. Chem. Soc. 1992, 114, 6761.

8. (a) Gram, D. Science 1988, 240, 760; (b) Lehn, J. M. Angerw. Chem. Int. Ed. Engl 1988, 27, 89.

9. (a) Cooks, R. G.; Kruger, T. L. I. Am. Chem. Soc. 1977, 99, 1279-1281; (b) McLuckey, S. A.; Cameron, D.; Cooks, R. G. J. Am. Chem. Soc. 1981, 103, 1313-1317; (c) Graul, S. T.; Schnute, M. E.; Squires, R. R. Int. J. Mass Speclrom. Ion Processes 1990, $96,181-198$.

10. Uppal, J. S.; Staley, R. H. J. Am. Chem. Soc. 1982, 104, 1235.

11. Lias, S. G.; Liebman, J, F, Levin, R. D. T. Phys. Chem. Ref. Data $1984,13,695$

12. Solomon, J. J.; Porter, R. F. J. Am. Chem. Soc. 1972, 94, 1443.

13. Munson, M. S. B.; Field, F. H. I. Am. Chem. Soc. 1965, 87, 3294.

14. Beauchamp, J. L.; Holtz, D.; Woodgate, S. D.; Patt, S. L. J. Am. Chem. Soc. 1972, 94, 2798.

15. Sharma, R. B.; Blades, A. T.; Kebarle, P. I. Am. Chem. Soc. 1984, 706, 510-516.

16. Basic, C.; Eyler, J. R.; Yost, R. A. I. Am. Soc. Mass Spectrom. 1992, 3, 716 . 